\title{
Discrimination of Buried Objects using Time-Frequency Analysis and Waveform Norms
}

\author{
Ivor L. Morrow*, Sebastian Wirth*, Mark Finnis* \\ ${ }^{*}$ Cranfield University, Laboratory of Electromagnetic Systems Engineering, \\ Shrivenham Campus, Oxfordshire, SN6 8LA, UK. \\ Email: i.l.morrow@cranfield.ac.uk
}

\begin{abstract}
Ground Penetrating Radar (GPR) are widely used to probe the sub-surface. Recently, various time-frequency analyses has been proposed to discriminate buried land mines from other clutter objects and thus reduce GPR false alarm rates. This paper examines the possibility for discrimination and assesses it experimentally. The approach uses the Choi-Williams timefrequency transform to analyse ultra-wideband signal returns from a range of shallow buried objects. Single Value Decomposition is performed on isolated object time-frequency signatures. The signatures are evaluated using a set of waveform norms that discriminate in time, frequency and energy content. The results indicate that this approach could improve land mine detection rates and reduce false alarms.
\end{abstract}

Keywords: - time-frequency transforms, remote sensing, signal theory and analysis.

\section{INTRODUCTION}

Typically land mine discrimination, i.e. detection and possibly identification, is achieved using synthetic aperture processing (SAR) techniques and image processing algorithms [1], such approaches are computationally intensive and require a trained operator [2]. Detection of non-metallic (NM) and minimum metal (MM) anti-personnel land mines (APLs) with ground penetrating radar (GPR) is often made difficult because of the clutter environment within the first $5 \mathrm{cms}$ of soil surface [3]. On the other hand most APLs are placed within that region to ensure reliable detonation when trod on.

In the literature the benefits of representing wideband radar data in the time-frequency domain has been recognised and applied in various ways for the purpose of target signature identification [4]. Capturing the scattering properties of a target such as resonances, dispersive propagation, creeping waves and broadband scatterers results in a signal that has greater information content than the relatively low resolution product obtained from SAR [5] imagery. In this paper the ultrawideband GPR returns of various buried objects including land mines are measured. A bi-linear time-frequency transform is applied to the radar echogram (or A-scan). This is decomposed into a time-frequency (two-dimensional) representation where the localisation of an object and its scattering behaviour can be analysed.

land mine detection techniques using Short-Time FourierTransform (STFT), Wigner-Ville Distribution (WVD), Radon Wigner Distriibution (RWD) and the S-Transform have been reported (see [6] for an overview). Lopera [7] pointed out that signatures from WVD contain more valuable information than that extracted using Wavelet Transform (WT). Sun et al [8]

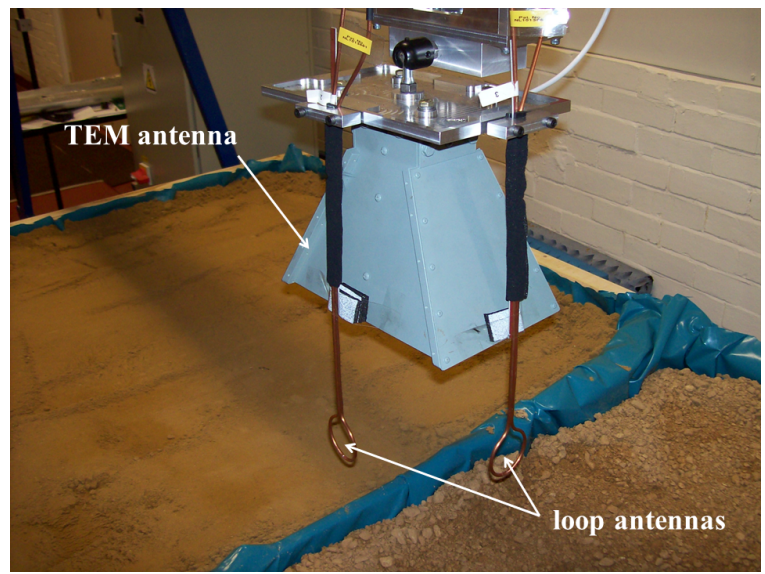

Fig. 1: Photograph of the assembled antenna set-up used in ground penetrating radar experiments. A TEM horn illuminates the ground in vertical $(\mathrm{V})$ polarisation. Two loop antenna are arranged orthogonally to pick up the backscattered $\mathrm{VV}$ and VH co-and cross-polarised signals.

also focused on the comparative results and their conclusion was the Choi-Williams Distribution (CWD) provide better discrimination results than STFT and other smoothed WVD.

The paper is organised as follows: In Section II the ground penetrating radar set up and test environment are described along with the signal pre-processing that is applied to minimise noise and clutter effects. Section II-B describes the properties of the Choi-Williams distribution or transform and its practical application to UWB GPR data. Single Value Decomposition (SVD) is then applied to extract eigenvalues and vectors from which a set of waveform norms are defined and used to analyse the time frequency and energy content of various buried objects. The results are plotted in three-dimensional space and demonstrate the technique. Section III provides an assessment on the techniques effectiveness.

\section{GPR DATA ACQUISITION AND PROCESSING}

\section{A. Experimental Set-up}

Initial experiments have been conducted on the indoors soil facility which consists of three soil bays (each 1.2 Lx $1.2 \mathrm{Wx} 0.8 \mathrm{H}$ meters) filled with different soils types; sand, a 50:50 by \% sand/shale mix and local Down Ampney peaty loam. The soils are maintained within a 3-8\% moisture content and at a constant temperature of $25^{\circ} \mathrm{C}$. A Near-Field 


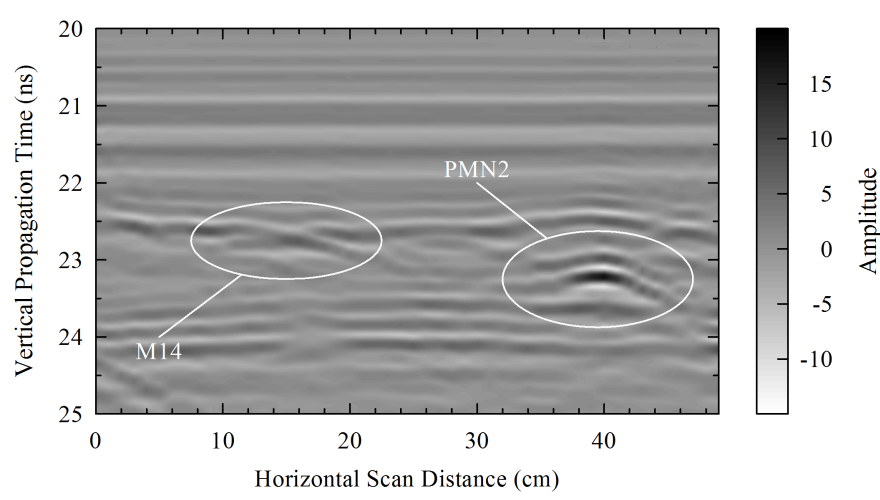

Fig. 2: A processed B-scan of some buried objects in dry sand including two surrogate land mine a PMN-2 buried $10 \mathrm{cms}$ below surface and an M14 buried $3 \mathrm{~cm}$ below the surface.

Measurement System (NFMS) has been erected over the three bays with a $3.5 \mathrm{~m}$ long linear automated positioner. The antenna array acquires the scattering parameter $S_{21}$ across the 0.4-5.0 GHz frequency spectrum and takes measurements at $1 \mathrm{~cm}$ intervals along the horizontal $\mathrm{x}$-axis. Figure 1 shows the dual polarised near-field antenna configuration. The setup uses a linear polarised TEM (Scientific Atlanta) horn that illuminates the ground scene in vertical $(\mathrm{V})$ polarisation. The local back-scattered electromagnetic field is collected by two loop antennas arranged orthogonally and in front of the TEM antenna to acquire VV and VH (vertical-horizontal) co- and cross-polarised data. The loop antenna are $30 \mathrm{cms}$ above the soil surface. The antenna height is typical of a stand-off GPR. This height is a trade-off between such factors as the energy attenuation, decreasing the antenna-soil coupling, avoiding possible obstacles of the ground surface, and forming sufficient antenna footprint for the SAR processing. The loop antennas are located just outside the near-eld of the TEM horn, at $11 \mathrm{~cm}$ in front of the aperture at $400 \mathrm{MHz}$. Figure 2 shows a B-scan made using this antenna set-up over the sand bay where several buried objects can be clearly seen. Background subtraction has been applied to remove any stationary artefacts present such as mutual coupling and air-ground interface reflections. Using this antenna set up a number of UWB scans were acquired with different shallow buried land mines and clutter objects present in the sand. The data from these experiments are used in the remainder of the paper.

\section{B. Time-Frequency Analysis}

The usefulness of the joint time-frequency analysis of signals has long been recognized in the signal processing arena [9]. Perhaps the most well known and used transform is the Wigner-Ville distribution (WVD). This quadratic or bilinear transform can be shown to provide better time frequency resolution compared to linear transforms such as the ShortTime Fourier transform (STFT) or the continuous Wavelet Transform. An important property of the WVD are independence of time, frequency, or phase shift and preservation of the signal energy. A serious limitation of the WVD is cross term interference for multiple signals. This can hinder the usefulness of the WVD for detecting signal characteristics in the timefrequency plane.
More generally, other types of time-frequency distributions such as the Choi-Williams distribution (CWD) can be designed to reduce the cross-term interference while preserving the WVD properties. The general form of the $C W D(t, \omega)$ of an A-scan $s(t)$ signal may be given as,

$$
\begin{array}{r}
C W D(t, \omega)=\iint \phi\left(t, t^{\prime}\right) s\left(u+\frac{t^{\prime}}{2}\right) s^{*}\left(u-\frac{t^{\prime}}{2}\right) \\
\times \exp \left(-j \omega t^{\prime}\right) d u d t^{\prime}
\end{array}
$$

where $\phi\left(t, t^{\prime}\right)$ is a kernel function designed to reduce the cross-term interference problem. It is essentially a low pass filter where $\alpha$ is set to one.

$$
\phi\left(t, t^{\prime}\right)=\frac{1}{\sqrt{4 \pi \alpha\left(t^{\prime}\right)^{2}}} \exp \left(-\frac{(t-u)^{2}}{4 \alpha\left(t^{\prime}\right)^{2}}\right)
$$

Where $s(t)$ is the analytic signal, $u=t f_{s}$, and $f_{s}$ is the sampling frequency. This transforms the one-dimension signal to the resultant (magnitude) $|C W D(t, \omega)|$ two-dimensional representation. It is usual to apply some form of windowing such as a Hanning or as here a rectangular window is applied [9].

\section{Feature Extraction}

Singular values of the CWD may be extracted using the SVD technique [10]. The CWD matrix $W$ s with its singular values and singular vectors, are unique for any matrix:

$$
W_{s}[m, n]=U[m, n] \Sigma[m, n] V[m, n]
$$

Where, the matrix $U$ and $V$ contain the left-singular and right-singular vectors, respectively, and the matrix $\Sigma$ contains the singular values. Namely, any matrix can be decomposed into a number of singular triplets $\mathbf{u}_{\mathbf{k}}, \sigma_{\mathbf{k}}, \mathbf{v}_{\mathbf{k}}$, with $k=$ 1.. $\min (M, N)$, where each singular value $\sigma_{k}$ can be treated as the square root of the corresponding triplet energy. The rows and columns of $W_{s}$, represent the distribution of the energy in time and frequency, respectively. The singular values $\mathbf{v}_{\mathbf{k}}$ and $\mathbf{u}_{\mathbf{k}}$ can be treated as the time and frequency representations of the CWD.

The signature decomposition is demonstrated in Fig. 3. The original time-frequency signature is shown top-left for an Ascan acquired directly over a buried PMN-2 surrogate land mine. The first singular component is the strongest, and it creates the high-energy part of the time frequency signature, i.e., the most robust part, is shown in the signature topright. It can be interpreted as a signature approximation, while the second component expresses the next important signature detail, shown bottom-left. The ideal image signature for the given land mine in this case is the composite $(1 \mathrm{st}+2 \mathrm{nd})$ modes shown bottom-right. Higher order singular components (the third and higher) could also be considered as discriminator features. However, they show significant change due to any small modification of the physical scan or due to any change of the target response. We do not include these transitory components in further analysis. 


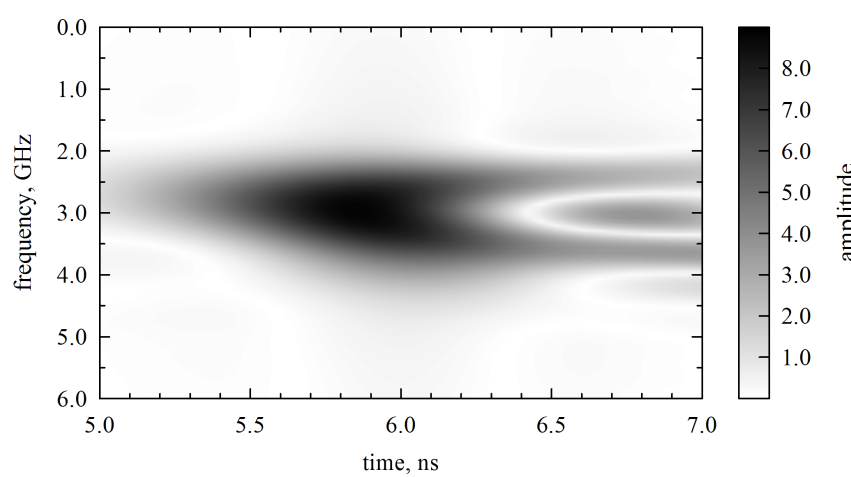

(i) Original for PMN-2

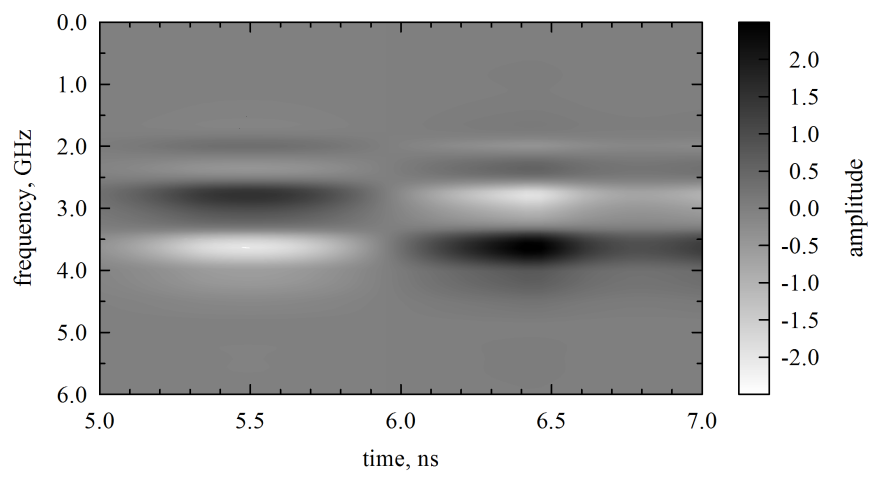

(iii) 2nd singular component

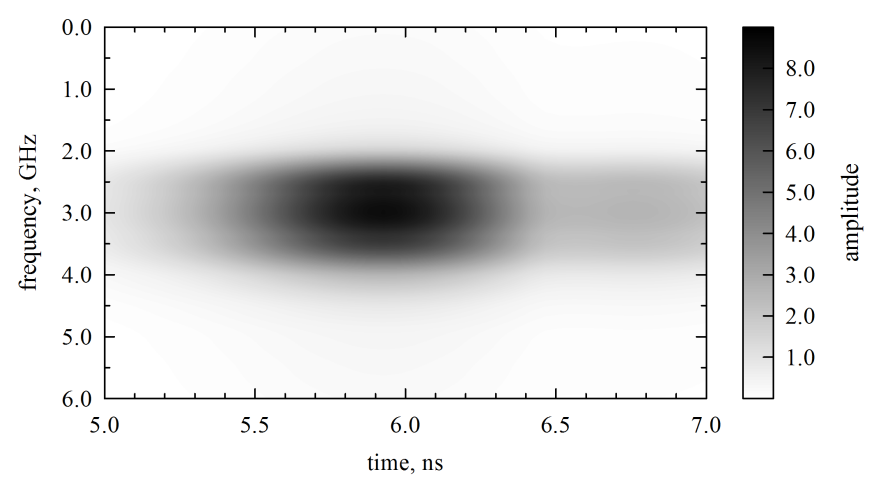

(ii) 1st singular component

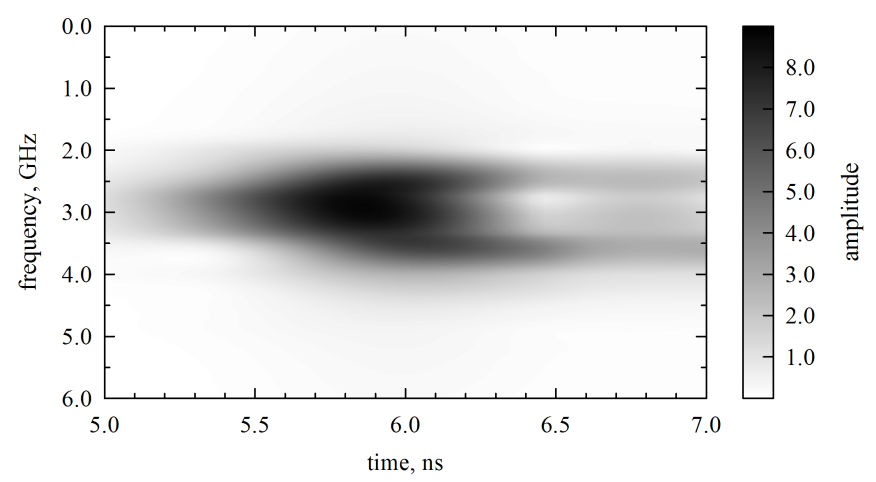

(iv) $1 \mathrm{st}+2 \mathrm{nd}$ singular components

Fig. 3: Approximation of CWD by the principal singular components.

Waveform norms are a branch of signal analysis used in EMC/EMI testing to characterise the properties of an arbitrary $\mathrm{RF}$ waveform. It was suggested in [7] that some of these norms for energy, time and frequency characteristics could be used as discriminators. Since the centre-of-gravity (CoG) of a two-dimensional array is the most robust point in any distribution. We use this concept combined with the SVD to extract eigenvalues and discriminate the more robust stationary scattering time-frequency features. The following normalized feature set was adopted since it is shown they can provide good discrimination results [8].

In total, we may extract six parameters from the CWD, namely $t_{1}, t_{2}, f_{1}, f_{2}, \sigma_{1}$ and $\sigma_{2}$ norms. These parameters could be used as target features directly. However, the signal duration and bandwidth are inter-related viz. the time-bandwidth $(=1.0)$ product. We can reduce the dimensionality of the feature space by multiplying the parameters $t_{k}$ and $f_{k}$ for the same principal component. Leading to a compact form of timefrequency-norm products or "feature set" as defined below,

$$
\begin{array}{r}
\delta_{t}=\frac{1}{N} \Sigma_{n=1}^{N} n v_{1}[n]^{2} \\
\delta_{f}=\frac{1}{M} \Sigma_{m=1}^{m} m u_{1}[m]^{2} \\
\delta_{e}=\frac{\sigma_{1}}{\Sigma_{k=1}^{\min [M, N]} \sigma_{k}^{2}}
\end{array}
$$

Where $\delta_{t}, \delta_{t}$, and $\delta_{t}$ are the time, frequency and energy features. Ideally, one anticipates a dense cluster of such points for the same target, and such clusters should ideally be well separated from each other for different targets. Fig. 4 shows the plotted feature extracted from five A-scans taken over the back scatter hyperbola for the PMN-2 surrogate land mine. Taking more A-scans around the surrogate land mine provided more observation vector plots. We observe, from Fig. 4, a good clustering in feature space.

Fig. 5 shows the extracted feature set $\delta_{t}, \delta_{f}$, and $\delta_{e}$ for two surrogate land mines; an M14 (56x40 mm in size) and a PMN-2 (115x57 mm in size) and a range of other buried clutter objects including dielectric plates of relative permittivity 5 and 15 (both approximately 100x100x10 mm in size) and a crushed coke can $(115 \times 65 \times 3 \mathrm{~mm}$ in size). The APLs targets and clutter objects are shallow buried in the sand, typically $\leq 10 \mathrm{cms}$. To increase the number of observation features plotted a vector of A-scan over the top of an object has been processed. It can be seen from Figure 5 that different objects are well clustered with some degree of separation between the clusters.

In Figure 5 the M14 and PMN-2 land mines appear clustered and fairly close in feature space. Since they possess similar construction materials and dimensions this is not unexpected. The two dielectric blocks show distinctly differing feature sets. Resonant frequency features are similar but time and energy characteristics differ markedly. The dielectric block of permittivity 15 possess a weaker backscatter energy feature. This is due to a stronger dielectric resonator action (and 


\section{PMN2}

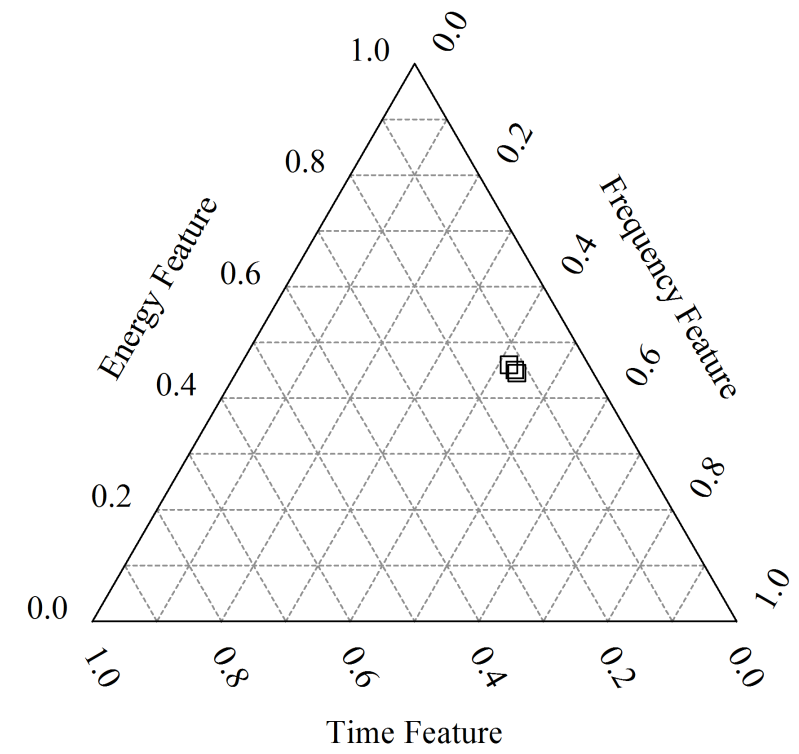

Fig. 4: Features extracted from singular feature vectors of CWD for five A-scans over the PMN-2 land mine.

higher Q-factor) that the lower dielectric constant block. It was anticipated that the dielectric block of permittivity 5 would be close to the M14 and PMN-2 feature sets and this is so. The coke can is metallic clutter and could potentially present a series of false alarms to a de-mining radar. Since it presents a surprisingly well clustered feature set that is also close to that of the lower dielectric constant block.

\section{DisCUSSION OF RESULTS}

A technique has been proposed to enable detection and accurate discrimination of buried land mines from other buried objects. It relies on GPR UWB waveform illumination, background subtraction and analysis of the singular values and vectors of the CWD. The CWD of an isolated object are unique to the target and related to the dielectric constant, its geometric design and polarisation of the incident field. An optimum set of waveform norm features were identified and used to characterise various buried objects. Plots of the threedimensional feature sets illustrate the potential to discriminate different types of buried land mines and land mines from clutter. Further work is currently under way considering different ground types, moisture conditions, and antenna configurations.

\section{ACKNOWLEDGMENT}

The authors thank the Find A Better Way charity for their support of this research under the DETERMINE programme (grant number 2015/001D).

$\begin{array}{ll}\square \text { Coke Can } & \triangle \text { Dielectric Plate 15 } \\ \nabla \text { Dielectric Plate 5 } & \triangle \text { M14 } \\ & \square \text { PMN-2 }\end{array}$

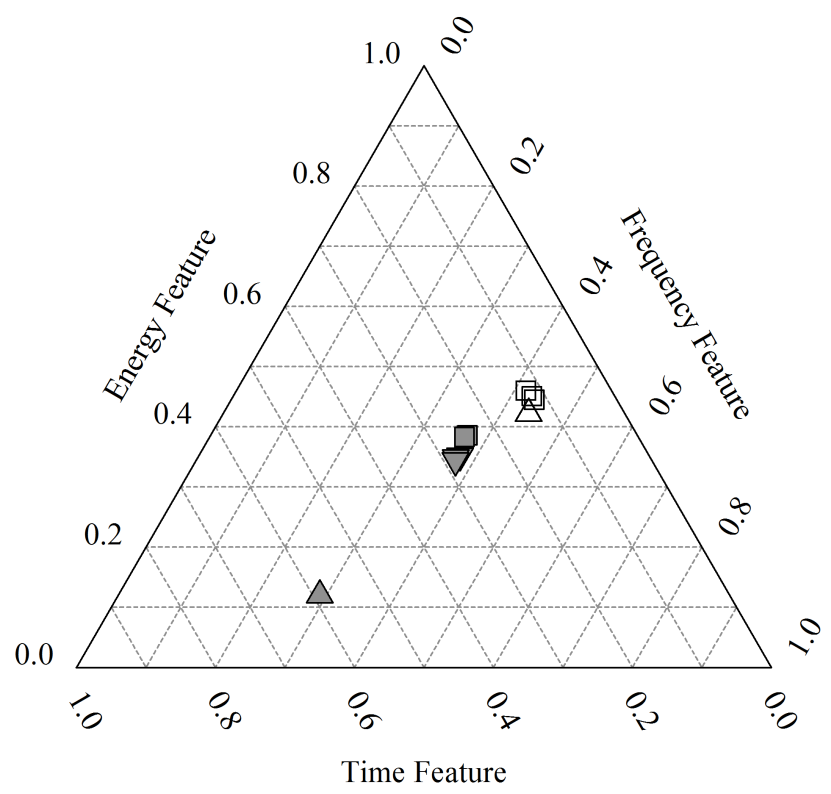

Fig. 5: Features extracted from singular feature vectors of the CWD for shallow buried APLs and some clutter objects.

\section{REFERENCES}

[1] I. Morrow, "Reconstruction algorithm for object determination from ultra-wideband field data," ICAP, vol. 40, no. 2, pp. 812-815, 31st March-3rd April 2003.

[2] I. Morrow and P. van Genderen, "Effective imaging of buried dielectric objects," Geoscience and Remote Sensing, IEEE Transactions on, vol. 40, no. 4, pp. 943-949, 20022002.

[3] B. Wong, I. Chant, G. Crisp, K. Kappra, K. Sturgess, A. Rey, and K. Sherbondy, "Suggested soil characterization techniques and surrogate targets for ultra-wide-band radar mine detection experiments," SPIE, vol. Vol. 3079, pp. 18-23, 1997.

[4] A. Papandreou-Suppappola, Applications in Time-Frequency Signal Processing, 1st ed., ser. Electrical Engineering and Applied Signal Processing Series. CRC Press, 2002.

[5] T. Savelyyev, L. Kempen, and H. Sahli, "Investigation of time-frequency features for gpr landmine discrimination," Geoscience and Remote Sensing, IEEE Transactions on, vol. 45, pp. 18-23, July 2007.

[6] D. Daniels, Ground Penetrating Radar, 2004, vol. Institution of Electronic and Technology, 2nd Edition.

[7] O. Lopera, "Time-frequency domain signature analysis of gpr data for landmine identification," IEEE Advanced Ground Penetrating Radar Workshop, Nice, 2007.

[8] Y. Sun and J. Li, "Time-frequency analysis for plastic landmine detection via forward-looking ground penetrating radar," IEE Proc. Radar Sonar Navigation, vol. 150, August 2003.

[9] V. C. Chen, Time-Frequency Transforms for Radar Imaging and Signal Analysis, 1st ed., ser. Artech House radar library. Artech House, 2002.

[10] S. V. Teukolsky, W. T. Vetterling, and B. P. Flannery, "Numerical Recipes in Fortran," 1994. 